\title{
Pembrolizumab- and ipilimumab-induced diabetic ketoacidosis and isolated adrenocorticotropic hormone deficiency: a case report
}

Thachanun Porntharukchareon ${ }^{1}$, Borwonkhun Tontivuthikul ${ }^{2}$, Nattaya Sintawichai ${ }^{3}$ and Panudda Srichomkwun ${ }^{2,4^{*}}$

\begin{abstract}
Background: Several human monoclonal antibodies directed against immune checkpoints, including T lymphocyte antigen 4 and programmed cell death protein 1, have been implemented for cancer treatment in order to promote effector $\mathrm{T}$ cell response to tumors. Despite the antitumor activity of these agents, a significant number of patients demonstrated immune-related adverse events that affected the functions of multiple organs, including the endocrine system. We report the first case of immune checkpoint inhibitor-induced simultaneous diabetic ketoacidosis and isolated adrenocorticotropic hormone deficiency following combination treatment with immune checkpoint inhibitors.

Case presentation: A 70-year-old Thai man with no previous history of diabetes mellitus was diagnosed with stage IVB non-small cell lung with pleural and liver metastases. After 14 weeks of combination treatment with pembrolizumab and ipilimumab, he presented with fatigue, nausea, and vomiting. Laboratory investigation revealed random plasma glucose $794 \mathrm{mg} / \mathrm{dl}$, serum ketone $6.3 \mathrm{mmol} / \mathrm{L}$, bicarbonate $13 \mathrm{mmol} / \mathrm{L}$, and high anion gap $24 \mathrm{mmol} / \mathrm{L}$. New-onset diabetes mellitus and diabetic ketoacidosis were diagnosed. Insulin therapy was initiated a favorable outcome within 10 hours. Despite improvement of hyperglycemia, the patient had persistent nausea and hyponatremia. Further investigation revealed cortisol $0.8 \mu \mathrm{g} / \mathrm{dl}$ and adrenocorticotropic hormone $21.7 \mathrm{pg} / \mathrm{ml}$. His other pituitary hormone levels were normal, except for mild elevation of gonadotropin hormone. Magnetic resonance imaging of the pituitary showed a normal pituitary gland. Isolated adrenocorticotropic hormone deficiency was diagnosed, and corticosteroid replacement therapy was administered, resulting in an improvement of his symptoms.
\end{abstract}

Conclusion: Our patient developed new-onset diabetes mellitus, diabetic ketoacidosis, and isolated adrenocorticotropic hormone deficiency during cancer treatment with pembrolizumab and ipilimumab. The present case highlights the need for physicians to be aware that immune-related adverse events can occur in multiple organs at the same time.

Keywords: Immune-related adverse event, Diabetic ketoacidosis, Isolated adrenocorticotropic hormone deficiency, Immunotherapy, Immune checkpoint inhibitors

\footnotetext{
* Correspondence: panuddas@hotmail.com

2Division of Endocrinology and Metabolism, Department of Medicine, Faculty of Medicine, Chulalongkorn University and the King Chulalongkorn Memorial Hospital, Bangkok, Thailand

${ }^{4}$ Excellent Center in Diabetes, Hormones and Metabolism, King Chulalongkorn Memorial Hospital, Thai Red Cross Society, Bangkok, Thailand Full list of author information is available at the end of the article
} 


\section{Background}

The modern era of cancer treatment is constantly evolving, with new breakthroughs and discoveries. Immune checkpoint inhibitors (ICIs) are a new and effective class of cancer immunotherapy. Several human monoclonal antibodies directed against immune checkpoints, including cytotoxic T-lymphocyte-associated protein 4 (CTLA$4)$, program death protein 1 (PD-1), and programmed death-ligand 1 (PD-L1), have been implemented for cancer treatment in order to promote an effector $\mathrm{T}$ cell response to tumors. Despite the antitumor activity of these agents, a significant number of patients demonstrated autoimmunity leading to immune-related adverse events (IRAEs). IRAEs can potentially affect functions of multiple organs, including the endocrine system. Hypophysitis, thyroid dysfunction, insulin-deficient diabetes mellitus (DM), and primary adrenal insufficiency have been reported as IRAEs due to ICI therapy [1]. Insulin-deficient DM and adrenal insufficiency are infrequent ICI-related endocrinopathies but can result in life-threatening diabetic ketoacidosis (DKA) or adrenal crisis, respectively, without early diagnosis and appropriate management. In this report, we present a case of ICI-induced simultaneous DKA and isolated adrenocorticotropic hormone deficiency (IAD) following combination treatment with pembrolizumab and ipilimumab in a patient with advanced non-small cell lung cancer (NSCLC).

\section{Case presentation}

A 70-year-old Thai man was diagnosed with stage IVB NSCLC with pleural and liver metastases. The patient's medical history was unremarkable until December 2018, when he had right pruritic chest pain and nonproductive cough. The patient had smoked cigarettes (20 packs per year) and did not have a drinking habit. The patient had good performance status, and he was not taking any medications. He was found to have high levels of PD-L1 expression (tumor proportion score $\geq 50 \%$ ) but had a negative result for epidermal growth factor receptor mutations and anaplastic lymphoma kinase rearrangement. He was treated with a combination of pembrolizumab $200 \mathrm{mg}$ every 3 weeks and ipilimumab $1 \mathrm{mg} / \mathrm{kg}$ every 6 weeks. A combination of PD- 1 and CTLA-4 inhibitors was used as a first-line treatment in this patient because the available evidence suggested that combination therapy may produce a higher tumor response rate than PD1 inhibitors alone in metastatic NSCLC PD-L1 positive subgroups [2].

After 14 weeks of treatment, the fifth cycle of pembrolizumab and the third cycle of ipilimumab, he presented with complaints of fatigue, vigorous nausea, and vomiting without abdominal pain. His physical examination revealed his body temperature was $37.2^{\circ} \mathrm{C}$, blood pressure was $100 / 60 \mathrm{mmHg}$, pulse rate was 116 beats per minute, and respiratory rates was 20 breaths per minute. The examination of his abdomen, neurological system, and other systems was unremarkable.

The patient's random plasma glucose level was 794 $\mathrm{mg} / \mathrm{dl}$, and his serum ketone level was $6.3 \mathrm{mmol} / \mathrm{L}$. His arterial blood gas analysis showed a $\mathrm{pH}$ of 7.17. He was admitted to the hospital, and the results of further laboratory investigations are shown in Table 1. Hyperglycemia, high serum ketone, low bicarbonate at $13 \mathrm{mmol} /$ $\mathrm{L}$, and high anion gap at $24 \mathrm{mmol} / \mathrm{L}$ were compatible with the DKA criteria. A treatment protocol for DKA with aggressive intravenous hydration and continuous intravenous insulin was initiated with a favorable outcome within 10 hours.

The diagnosis of ICI-related DM was suspected due to the abrupt onset of DM. Blood analysis revealed an undetectable C-peptide level and a negative result of glutamic acid decarboxylase autoantibodies (anti-GAD) and anti-tyrosine phosphatase-like islet antigen 2 .

Despite his hyperglycemia improving, he still had persistent nausea and hyponatremia (serum sodium 126 $\mathrm{mmol} / \mathrm{L})$. Further investigations revealed a very low morning cortisol level $(0.8 \mu \mathrm{g} / \mathrm{dl})$ and normal adrenocorticotropic hormone $(\mathrm{ACTH})$ level $(21.7 \mathrm{pg} / \mathrm{ml}$; normal range $0-46)$. His other pituitary hormone levels were normal, except for mild elevation of follicle-stimulating hormone/luteinizing hormone (Table 1). He was diagnosed with IAD and immediately received intravenous hydrocortisone. Magnetic resonance imaging (MRI) of the pituitary showed a normal pituitary gland (Fig. 1).

Twenty-four hours after starting corticosteroid replacement, his symptoms and hyponatremia resolved. He was then switched from hydrocortisone to prednisolone. He was discharged on the 12th day of admission with prednisolone $7.5 \mathrm{mg} /$ day and premixed insulin 26 U/day. Both his pembrolizumab and ipilimumab were discontinued due to IRAEs.

Six months following hospital admission, he was seen in regular follow-up in the endocrinology department. His blood glucose levels were borderline controlled with premixed insulin $58 \mathrm{U} /$ day (Table 1 ). He remained on prednisone $7.5 \mathrm{mg} /$ day, and he felt extremely fatigued if he missed a dose of prednisolone. He had stable disease even after ICI discontinuation and no further treatment.

\section{Discussion}

ICIs are approved for many types of cancer. Pembrolizumab is a PD-1 inhibitor approved for melanoma, NSCL $\mathrm{C}$, small cell lung cancer, renal cell carcinoma, and head and neck squamous cell carcinoma. Ipilimumab is a CTLA-4 inhibitor approved for melanoma, renal cell carcinoma, microsatellite instability-high, or mismatch repair deficiency colorectal cancer [1]. Combination therapy with CTLA-4 inhibitors and PD-1/PD-L1 
Table 1 Laboratory data of the patient

\begin{tabular}{|c|c|c|c|c|}
\hline Investigation & 1 month before admission & This admission & 6 months after admission & Reference range \\
\hline Plasma glucose, mg/dl & 87 & 794 & 210 & 70-99 \\
\hline $\mathrm{HbA} 1 \mathrm{C}, \%$ & N/A & 6.5 & 7.0 & $4.0-5.6$ \\
\hline Beta-hydroxybutyrate, mmol/L & N/A & 6.3 & N/A & $0-0.6$ \\
\hline Sodium, mmol/L & 135 & 119 & 140 & $136-145$ \\
\hline Potassium, mmol/L & 3.3 & 6.5 & 3.4 & $3.4-4.5$ \\
\hline Chloride, $\mathrm{mmol} / \mathrm{L}$ & 97 & 82 & 99 & $95-105$ \\
\hline Bicarbonate, $\mathrm{mmol} / \mathrm{L}$ & 28 & 13 & 26 & $22-29$ \\
\hline Anion gap, mmol/L & 10 & 24 & 15 & $8-16$ \\
\hline Blood urea nitrogen, mg/dl & 5 & 39 & N/A & $7-20$ \\
\hline Creatinine, mg/dl & 0.75 & 1.8 & 0.82 & $0.7-1.2$ \\
\hline Amylase, U/L & N/A & 60 & N/A & 20-100 \\
\hline Lipase, U/L & N/A & 53 & N/A & $10-60$ \\
\hline Anti-GAD, IU/ml & N/A & 3.09 & N/A & $<17$ \\
\hline Anti-IA2, IU/ml & N/A & $<7.5$ & N/A & $<7.5$ \\
\hline C-peptide & N/A & $<0.1$ & N/A & $0.9-7.1$ \\
\hline 8:00 a.m. cortisol, ng/ml & N/A & 0.6 & N/A & $3-18$ \\
\hline $\mathrm{ACTH}, \mathrm{pg} / \mathrm{ml}$ & N/A & 21.7 & N/A & $0-46$ \\
\hline $\mathrm{FT} 4, \mathrm{ng} / \mathrm{dl}$ & N/A & 1.22 & 1.19 & $0.8-1.8$ \\
\hline $\mathrm{TSH}, \mu \mathrm{lU} / \mathrm{ml}$ & N/A & 2.94 & 2.560 & $0.3-4.1$ \\
\hline FSH, IU/L & N/A & 17.7 & 13.9 & $1-8.4$ \\
\hline $\mathrm{LH}, \mathrm{IU} / \mathrm{L}$ & N/A & 19.4 & 8.1 & $1-10.5$ \\
\hline Testosterone, $\mathrm{nmol} / \mathrm{L}$ & N/A & 24.46 & 14.89 & $5.9-24.7$ \\
\hline IGF-1, ng/ml & N/A & $\mathrm{N} / \mathrm{A}$ & 66.4 & $24.6-269$ \\
\hline Prolactin, ng/ml & N/A & 15.9 & 6.7 & $2-25$ \\
\hline
\end{tabular}

Abbreviations: ACTH Adrenocorticotropic hormone, FSH Follicle-stimulating hormone, FT4 Free thyroxine, GAD Glutamic acid decarboxylase, IA2 Islet antigen 2, IGF1 Insulin-like growth factor 1, LH Luteinizing hormone, N/A Not available, TSH Thyroid-stimulating hormone Boldface define abnormal value Boldface define abnormal value

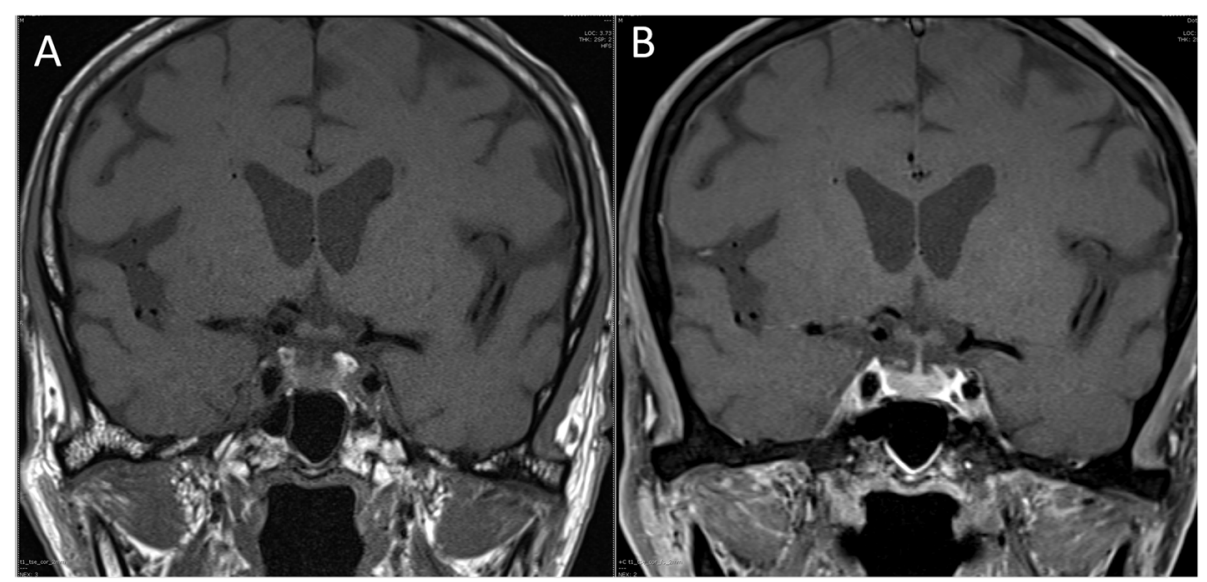

Fig. 1 Magnetic resonance imaging (MRI) of the pituitary gland, coronal plane. a T1-weighted MRI showing no abnormalities in the pituitary gland, hypophyseal stalk, and hypothalamus. b Gadolinium-enhanced T1-weighted MRI showing a symmetric, round-shaped pituitary gland with homogeneous enhancement of the hypophyseal stalk 
inhibitors have proved beneficial in advanced NSCLC, but they are limited by their serious side effects [2]. ICIrelated endocrinopathies resulting from combination therapy are hypothyroidism (5.6-22.5\%), hyperthyroidism (6.6-16.1\%), thyroiditis (3.8-4.6\%), hypophysitis (6.2-16.4\%), primary adrenal insufficiency (1.2-36.8\%), and DM (2\%) [3].

Our patient presented with DKA after the fifth cycle of pembrolizumab and the third cycle of ipilimumab with fulminant onset, undetectable C-peptide, and negative autoimmune DM antibodies. ICI-related DM is a rare but potentially life-threatening IRAE. Most ICIrelated DM cases have been due to PD-1/PD-L1 inhibitors rather than CTLA-4 inhibitors [1]. The incidence rates of $\mathrm{DM}$ in patients treated with only PD-1/PD-L1 inhibitors and a combination of PD-1/PD-L1 inhibitors plus CTLA-4 inhibitors are estimated at $1 \%$ and $2 \%$, respectively $[3,4]$.

Several important features characterize ICI-related DM: (1) abrupt onset of hyperglycemia, (2) rapid progression of endogenous insulin deficiency, and (3) high risk of DKA if not detected and treated promptly with insulin therapy [5]. De Filette et al. reported that DKA is the first clinical presentation in $71 \%$ of patients with ICI-related DM [5]. Autoimmunity is the main hypothesized pathogenesis of ICI-related DM; however, the conclusions remain unclear because half of the patients with ICI-related DM have a negative finding for autoimmune DM antibodies [1]. Time of presentation is inconsistent with a median time of onset of 3.1 cycles (range, 1-17) for patients with positive anti-GAD and 5.9 cycles (range, 1-16) for patients with negative anti-GAD findings, which is similar to the case of our patient [5]. Although the management of ICI-related DM is long-term insulin treatment, Hansen et al. reported one patient with ICI-related DM who was able to discontinue insulin and recovered from the C-peptide level after ICI discontinuation [6]. The limitation of our patient's case is the lack of information about genetic factors or human leukocyte antigen genotypes of the patient that may predispose to endocrine IRAEs [5, 7].

Several patients who developed ICI-related DM developed IRAEs prior to, concurrent with, or subsequent to the development of ICI-related DM, such as hypophysitis and thyroid dysfunction [1]. Our patient also has another endocrinopathy. He had a low cortisol level without elevated levels of ACTH and normal function of other pituitary axes, supporting a diagnosis of IAD.

ICI-related IAD is a rare endocrinopathy. Percik et al. reported that the prevalence of ICI-related IAD was $0.87 \%$ in their retrospective cohort study of all patients with cancer treated with ICI [8]. The risk for developing IAD was four times higher among women than among men and seven times higher in patients treated with combined PD-1/PD-L1 inhibitors plus ipilimumab than in patients treated with only PD-1/PD-L1 inhibitors (3.2\% vs. $0.4 \%$, respectively) [8]. In contrast to most autoimmune hypophysitis cases, IAD onset ranges from approximately 4 to 8 months, with a median period of 6 months, which appears to be longer than that for hypophysitis (approximately 2 months) [7]. Although the mechanism of the pathophysiology of ICI-related IAD has not been elucidated, the development of antipituitary autoantibodies and the direct effects of CTLA- 4 inhibitors on the pituitary are considered [9]. In contrast to conventional hypophysitis, the MRI findings of ICIrelated IAD mostly showed no enlargement of the pituitary gland, similar to the case of our patient [7]. The main treatment is long-term glucocorticoid replacement in a physiologic dose [8].

According to the latest version of the National Cancer Institute's Common Terminology Criteria for Adverse Events [10], our patient had a grade 4 adverse event for DKA and a grade 3 adverse event for ICI-related IAD. The treatment with ICI should be discontinued until $\mathrm{DM}$ is controlled and adequate hormone replacement therapy is administered [11]. ICI was discontinued in our patient due to IRAEs and poor performance status.

\section{Conclusion}

To the best of our knowledge, this is the first case report of ICI-induced DM/DKA and IAD following the combination treatment of PD-1 and CTLA-4 inhibitors. The present case highlights the need for physicians to be aware that IRAEs can occur in multiple organs at the same time.

\section{Abbreviations}

ACTH: Adrenocorticotropic hormone; CTLA-4: Cytotoxic T-lymphocyteassociated protein 4; DKA: Diabetic ketoacidosis; DM: Diabetes mellitus; FSH: Follicle-stimulating hormone; FT4: Free thyroxine; GAD: Glutamic acid decarboxylase; IA2: Islet antigen 2; IAD: Isolated adrenocorticotropic hormone deficiency; ICl: Immune checkpoint inhibitor; IGF-1: Insulin-like growth factor 1; IRAE: Immune-related adverse event; LH: Luteinizing hormone; MRI: Magnetic resonance imaging; N/A: Not available; NSCL C: Non-small cell lung cancer; PD-1: Programmed death 1; PDL1: Programmed death-ligand 1; TSH: Thyroid-stimulating hormone

\section{Acknowledgements \\ The authors thank the physicians of King Chulalongkorn Memorial Hospital who were involved in the management of our patient.}

\section{Authors' contributions}

TP, BT, NS, and PS contributed to patient management. TP was a major contributor to the writing of the manuscript. PS critically reviewed and revised the manuscript. All authors read and approved the final manuscript.

Funding

No sources of funding are declared for this study.

Availability of data and materials

All data generated during this study are included in this published article.

Ethics approval and consent to participate

Not applicable for this case report. 


\section{Consent for publication}

Written informed consent was obtained from the patient for publication of this case report and any accompanying images. A copy of the written consent is available for review by the Editor-in-Chief of this journal.

\section{Competing interests}

The authors declare that they have no competing interests.

\section{Author details}

${ }^{1}$ Chulabhorn Hospital, HRH Princess Chulabhorn College of Medical Science, Chulabhorn Royal Academy, Bangkok, Thailand. ${ }^{2}$ Division of Endocrinology and Metabolism, Department of Medicine, Faculty of Medicine, Chulalongkorn University and the King Chulalongkorn Memorial Hospital, Bangkok, Thailand. ${ }^{3}$ Division of Medical Oncology, Department of Medicine, Faculty of Medicine, Chulalongkorn University and the King Chulalongkorn Memorial Hospital, Bangkok, Thailand. ${ }^{4}$ Excellent Center in Diabetes, Hormones and Metabolism, King Chulalongkorn Memorial Hospital, Thai Red Cross Society, Bangkok, Thailand.

Received: 29 April 2020 Accepted: 17 August 2020

Published online: 29 September 2020

\section{References}

1. Chang LS, Barroso-Sousa R, Tolaney SM, Hodi FS, Kaiser UB, Min L. Endocrine toxicity of cancer immunotherapy targeting immune checkpoints. Endocr Rev. 2019:40(1):17-65.

2. Tanvetyanon T, Gray JE, Antonia SJ. PD-1 checkpoint blockade alone or combined PD-1 and CTLA-4 blockade as immunotherapy for lung cancer? Expert Opin Biol Ther. 2017;17(3):305-12.

3. de Filette J, Andreescu CE, Cools F, Bravenboer B, Velkeniers B. A systematic review and meta-analysis of endocrine-related adverse events associated with immune checkpoint inhibitors. Horm Metab Res. 2019;51(3):145-56.

4. Stamatouli AM, Quandt Z, Perdigoto AL, Clark PL, Kluger H, Weiss SA, et al. Collateral damage: insulin-dependent diabetes induced with checkpoint inhibitors. Diabetes. 2018;67(8):1471-80.

5. de Filette JMK, Pen JJ, Decoster L, Vissers T, Bravenboer B, Van der Auwera BJ, et al. Immune checkpoint inhibitors and type 1 diabetes mellitus: a case report and systematic review. Eur J Endocrinol. 2019;181(3):363-74.

6. Hansen E, Sahasrabudhe D, Sievert L. A case report of insulin-dependent diabetes as immune-related toxicity of pembrolizumab: presentation, management and outcome. Cancer Immunol Immunother. 2016;65(6):765-7.

7. Ohara N, Kobayashi M, Ohashi K, Ito R, Ikeda Y, Kawaguchi G, et al. Isolated adrenocorticotropic hormone deficiency and thyroiditis associated with nivolumab therapy in a patient with advanced lung adenocarcinoma: a case report and review of the literature. J Med Case Rep. 2019;13:88.

8. Percik R, Shlomai G, Tirosh A, Tirosh A, Leibowitz-Amit R, Eshet Y, et al. Isolated autoimmune adrenocorticotropic hormone deficiency: from a rare disease to the dominant cause of adrenal insufficiency related to check point inhibitors. Autoimmun Rev. 2020;19(2):102454.

9. Kitaijma K, Ashida K, Wada N, Suetsugu R, Takeichi Y, Sakamoto S, et al. Isolated ACTH deficiency probably induced by autoimmune-related mechanism evoked with nivolumab. Jpn J Clin Oncol. 2017:47(5):463-6.

10. National Cancer Institute. Common Terminology Criteria for Adverse Events (CTCAE). Version 5.0. November 27, 2017. https://ctep.cancer.gov/ protocoldevelopment/electronic_applications/docs/CTCAE_v5_Quick_ Reference_8.5x11.pdf. Accessed 1 Apr 2020.

11. Brahmer JR, Lacchetti C, Schneider BJ, Atkins MB, Brassil KJ, Caterino JM et al. Management of immune-related adverse events in patients treated with immune checkpoint inhibitor therapy: American Society of Clinical Oncology clinical practice guideline. J Clin Oncol. 2018;36(17):1714-68.

\section{Publisher's Note}

Springer Nature remains neutral with regard to jurisdictional claims in published maps and institutional affiliations.

Ready to submit your research? Choose BMC and benefit from:

- fast, convenient online submission

- thorough peer review by experienced researchers in your field

- rapid publication on acceptance

- support for research data, including large and complex data types

- gold Open Access which fosters wider collaboration and increased citations

- maximum visibility for your research: over $100 \mathrm{M}$ website views per year

At BMC, research is always in progress.

Learn more biomedcentral.com/submissions 